\title{
Recent advances in surface-enhanced Raman spectroscopy based liquid biopsy for colorectal cancer (Review)
}

\author{
LUCRETIA AVRAM $^{1}$, ANDREI STEFANCU $^{2}$, DANA CRISAN $^{3}$, NICOLAE LEOPOLD $^{2,4}$, VALER DONCA $^{1}$, \\ ELENA BUZDUGAN ${ }^{3}$, RARES CRACIUN $^{3}$, DAVID ANDRAS $^{5}$ and IOAN COMAN ${ }^{6}$ \\ ${ }^{1}$ Medical Specialities Department, 5th Medical Clinic, 'Iuliu Hatieganu' University of Medicine and \\ Pharmacy, 400012 Cluj-Napoca; ${ }^{2}$ Faculty of Physics, 'Babeș-Bolyai' University, 400084 Cluj-Napoca; \\ ${ }^{3}$ Internal Medicine Department, 5th Medical Clinic, 'Iuliu Hatieganu' University of Medicine and Pharmacy, \\ 400012 Cluj-Napoca; ${ }^{4}$ MEDFUTURE Research Center for Advanced Medicine, 'Iuliu Hatieganu' University of \\ Medicine and Pharmacy, 400349 Cluj-Napoca; ${ }^{5}$ Surgery Department, 1 st Surgery Clinic, and \\ ${ }^{6}$ Urology Department, 'Iuliu Hatieganu'University of Medicine and Pharmacy, 400012 Cluj-Napoca, Romania
}

Received August 28, 2020; Accepted September 29, 2020

DOI: $10.3892 /$ etm. 2020.9342

\begin{abstract}
As colorectal cancer (CRC) is one of the forms of cancer with the highest prevalence globally and with a high mortality, screening and early detection remains a major issue. Colonoscopy is still the gold standard for detecting premalignant lesions, but it is burdened by some complications. For instance, it is laborious, with some difficulties of acceptance for some patients, and is ultimately an imperfect standard, given that some premalignant lesions or incipient malignancies can be missed by colonoscopic evaluation. In this context, new non-invasive approaches such as surface-enhanced Raman spectroscopy (SERS) based liquid biopsy have gained ground in recent years, showing promising results in oncological pathology diagnosis. These new methods have enabled the detection of subtle molecular profile alterations prior to any macroscopic morphological changes, thus providing a useful tool for early CRC detection. In the present review, we provide a summary of published studies applying SERS in CRC detection, along with our personal experience in using SERS in the diagnosis of different oncological pathologies, including CRC.
\end{abstract}

Correspondence to: Professor Dana Crisan, Internal Medicine Department, 5th Medical Clinic, 'Iuliu Hatieganu' University of Medicine and Pharmacy, 8 Victor Babes Street, 400012 Cluj-Napoca, Romania

E-mail: crisan.dc@gmail.com

Dr Nicolae Leopold, Faculty of Physics, 'Babeș-Bolyai' University, 1 Mihail Kogalniceanu Street, 400084 Cluj-Napoca, Romania

E-mail: nicolae.leopold@phys.ubbcluj.ro

Key words: colorectal cancer, liquid biopsy, nanoparticles, Raman, SERS

\section{Contents}

1. Introduction

2. Methods and samples

3. Tumor biomarkers from body fluids targeted with SERS

4. Conclusions

\section{Introduction}

Colorectal cancer (CRC) is one of the most common types of cancer, ranking third in terms of prevalence among other primary sites, accounting for $11 \%$ of all cancer diagnoses and ranking second in cancer-related mortality (1). Its high prevalence has prompted large population scale screening programs, aiming at identifying patients with incipient forms of CRC in which curative therapeutic interventions are effective. For the moment, screening for CRC is recommended in asymptomatic adults aged 45-75 years. Patients with a high risk for developing CRC benefit from a specific screening protocol (family history of CRC, inflammatory bowel disease, known or suspected high risk genetic profiles). The standard target population for CRC screening consists in average risk patients, with none of the aforementioned characteristics (2). Screening in CRC is essential, as it detects the pre-cancerous structures that can develop in the colon, namely adenomatous polyps, but also early cancers. The gold standard for screening in CRC is colonoscopy, which was shown to decrease incidence by $64 \%$ [95\% confidence interval (CI) 50 to $74 \%$ ] and mortality by $66 \%$ (CI $38-81 \%$ ) in a meta-analysis of observational studies (3).

On the contrary, performing colonoscopy brings with it an important economic burden on healthcare systems, but also discomfort for patients and sometimes complications such as bleeding or perforation. Another drawback that comes frequently in the attention of gastroenterologists is that some premalignant lesions can be missed by standard endoscopy. For instance, in a study including 463 patients with 1294 neoplastic 
polyps being analyzed, the overall miss rate for adenomas was $24.1 \%(312 / 1294)$, while the miss rate for advance adenomas dropped to $1.2 \%$ (15/1294). No carcinomas were missed (0/1294). The higher missed rates were, as expected, for flat and small polyps (4).

Early detection of CRC is the main contributor to improving overall survival rates in this population, while saving costs for the medical system. Reliable (sensitive, specific and repeatable), cost-effective and rapid point-of-care methods based on liquid biopsy have the potential to greatly improve the early detection of any solid malignant tumor and it would highly reform the therapeutic approach. Consequently, patients could be dichotomized according to their screening results: a positive result should prompt further investigation, while negative results would be spared from further unnecessary and expensive tests. Liquid biopsy refers to the detection of molecular and cytological changes associated with cancer onset and early progression in biofluids such as blood, urine or saliva, thus bypassing the need for a tissue sample from the tumor. Liquid biopsy is usually performed using genomic, proteomic or metabolomics techniques (5). Raman spectroscopy was proven as a non-destructive and highly accurate cancer diagnostic tool (6-8), applied also for CRC detection (9). Surface-enhanced Raman spectroscopy (SERS) is a new emerging approach, which is sensitive for performing liquid biopsy and which gained a significant amount of interest due to its versatility (10-14).

In the present study, we provide a literature overview regarding SERS-based approaches in the detection of CRC and, also, our SERS results regarding the diagnosis of different types of oncological pathologies, including CRC.

\section{Methods and samples}

Raman spectroscopy and SERS-basic principles. Raman spectroscopy is in principle a vibrational spectroscopic method, in which molecular structural information is gained by analyzing the inelastic scattering of photons $(15,16)$. However, the technique suffers from low sensitivity, as only one in approximately 10 million photons is scattered inelastically, carrying thus Raman information. In 1974, a significant development was reported by Fleischman and Hendra: when pyridine molecules were adsorbed onto an electrochemically roughened silver electrode, a million-fold enhancement of the weak Raman signal was observed, exponentially increasing sensitivity when compared to the prior method (free molecules in a liquid environment) (17). The phenomenon, which came to be known as SERS, demonstrated a huge potential for analytical applications in various fields, including biomedicine (18-22). The Raman scattering amplification by plasmonic nanoparticles is explained in the literature by two distinct effects, the so called SERS electromagnetic theory (23) and the chemical effect (24).

The main advantages of SERS lay in the molecular specificity of Raman scattering and the high sensitivity of the method-comparable to that of fluorescence emission (20). Even SERS-based single molecule detection has successfully passed the sensitivity test (25). Furthermore, in case of biological samples with a strong auto fluorescence signal, which often hinders the recording of normal Raman spectra, the molecular adsorption to metal particles quenches the fluorescence emission, so that the SERS signal is not perturbed (26).

Despite SERS being a sensitive label-free highly molecular specific detection technique, the obtaining of the SERS signal is limited to molecules which adsorb to the metal nano-surfaces, so named molecules with surface-seeking groups, which ensure their adsorption onto the enhancing substrate (21). However, this disadvantage can be exploited in complex matrices such as blood serum or urine for selective analyte detection from complex matrices. Thus, albumin could be detected selectively in urine samples (27), or in case of SERS liquid biopsy for cancer detection, purine metabolites such as uric acid, xanthine or hypoxanthine have been shown to adsorb preferentially to silver or gold nanoparticle surfaces in blood serum or urine (10-14).

Recent fundamental studies indicate that the SERS spectra of cationic and anionic analytes are switched on in a specific manner by the type of adsorbed ion (adion) (28-30), the role of adions such as $\mathrm{Ag}^{+}, \mathrm{Ca}^{2+}, \mathrm{Mg}^{2+}, \mathrm{Cl}^{-}, \mathrm{Br}^{-}, \mathrm{I}^{-}$, being explained in the frame of the proposed adion-specific adsorption model (28). Thus, the SERS spectra of uric acid, xanthine and hypoxanthine purine metabolites are enhanced after activation of silver nanoparticles with cationic analytes such as $\mathrm{Ca}^{2+}$ or $\mathrm{Mg}^{2+}(11,12)$, whereas albumin and DNA detection require the activation of silver nanoparticles with $\mathrm{I}^{-}$and $\mathrm{Cl}^{-}$, respectively $(27,31)$.

Blood, plasma and serum samples. Peripheral blood samples are appropriate for liquid biopsy, as these can be obtained rapidly and non-invasively and the procedure can be repeated whenever needed during the diagnosis or follow-up. Blood is an essential biofluid which is comprised of a two-part mixture. The cellular part represents usually $\sim 40-50 \%$ of the total blood volume and contains red blood cells, white blood cells and platelets. On the other hand, plasma consists mainly of water $(>90 \%)$ and carries a large amount of proteins, mineral salts and organic compounds. Among them, there are thousands of metabolites, with an increasing number being identified at a rapid pace (so far exceeding 4600 identified metabolites) (32). Regarding plasma proteins, albumin is the most abundant, followed by coagulation factors, globulins involved in the immune system, enzymes, hormones and other specific proteins (32).

Because of the presence of cells, whole blood is not suitable for SERS analysis $(33,34)$. Therefore, only few reports deal with SERS analysis on whole blood, most studies targeting serum or plasma (35-38), serum being the part of plasma without coagulation factors (fibrinogens). SERS spectra of whole blood samples are dominated by resonant Raman bands of hemoglobin (39). However, the resonant Raman hemoglobin bands may be avoided by shifting the excitation laser wavelength from the blue-green region to the near infrared, at $785 \mathrm{~nm}$ (33).

\section{Tumor biomarkers from body fluids targeted with SERS}

Traditional biopsies suffer from intrinsic limitations related to precision and their invasive nature. They are not appropriate for early diagnosis, and often bring information concerning only a limited region of the tumor, especially in tumors 
with increased heterogeneity (40). Liquid biopsy can surpass these limitations of traditional biopsies by noninvasive rapid analysis of specific tumor biomarkers detected in circulating or excreted fluids. Thus, SERS-based liquid biopsy focuses on tumor-derived biomarkers such as circulating tumor cells (CTC), nucleic acids, proteins and exosomes.

Circulating tumor cells (CTC). An important challenge in the detection of CTCs is their low concentration, ranging from isolated molecules up to less than a thousand per $\mathrm{ml}$ of whole blood, compared to billions of other cells. Recent advances have led to the development of more sensitive microfluidic devices, able to isolate tumor cells in a highly efficient manner (41).

There are multiple approaches to SERS detection. Direct detection implies the direct adsorbtion of the species of interest to an active surface, whereas indirect detection requires the use of SERS-active labels, in a similar fashion to the use of fluorophores. An indirect SERS detection approach for CTC was reported by Pallaoro et al by using a platform that combines microfluidics with SERS (42). A mixture of prostate cells (both tumor and non-tumor) was incubated with spectral SERS rich tags, based on silver nanostructures labeled with a Raman-active small reporter molecule paired with an affinity biomolecule. The method was highly sensitive, reliably identifying passing tumor cells (among a significantly higher proportion of non-tumor cells), based on the specific Raman signatures. SERS spectroscopy was typically combined with different other techniques for the identification and measurement of multiplexed CTCs for the tumor tissue imagery and for fluorescence immunolabelling approaches $(43,44)$.

The CELLSEARCH System (Janssen Diagnostics) is the first and, as of now, the only FDA-approved device designed for colorectal, breast and prostate cancer automated CTC detection. This system selects the presumptive CTC using modified magnetic beads (using anti-epithelial cell adhesion molecule-EpCAM antibodies) (45-47). Other teams have designed dual-function magnetic-SERS nanoparticles. First, the iron oxide particles were coated with gold, then anti-EpCAM or anti-human epidermal growth factor receptor-2 (HER2) antibodies were added (48). These nanoparticles increase the sensitivity up to a few cells per $\mathrm{ml}$ and allow for on-line CTC magnetic separation and cell detection even in whole blood specimens.

The highly integrated magnetic-SERS nanoparticle cocktail allowed on-line magnetic separation and SERS detection of CTCs in whole blood, with a detection sensitivity down to $1-2$ cells/ml (48).

In order to fight the cost and excessive labor in binding antibodies, single-stranded oligonucleotides bound to different targets (called aptamers) were developed, which provide similar functions as antibodies. For example, a DNA KDED2a-3 aptamer binding DLD-1 cells (a colorectal adenocarcinoma cell line) was used for the magnetic labeling of nanoparticles and SERS tags. The sensitivity of the method was $73 \%$ from buffer and 55\% from whole blood samples (49). Individual cell multidimensional phenotyping of CTC might become a key contributor to future research in the field, providing diagnostic insights, as well as a therapeutic role by identifying potential targets, resistance pathways and prognostic assessment. Diversely, global analysis of the whole group of CTC generates a phenotypic signature which allows for treatment monitoring and might guide the therapeutic strategy (50).

In CRC, the SERS detection of CTCs was demonstrated in the case of 430 patients with metastatic disease. CTC detection was performed before treatment and during therapy, with the aim of demonstrating the prognostic utility of CTCs. The method used for the detection of CTCs was molecular immunomagnetic separation from patient's blood products before the commencement and during therapy. The results showed that unfavorable CTC before treatment was associated with a lower progression-free survival (51). Another study proved the detection of CTCs through a combination of magnetic capture and multiplex detection by using targeted magnetic nanoparticles and SERS probes (52).

Both SERS and magnetic nanoparticles are targeting the folate receptor. This receptor is typically overexpressed in cancer cells and absent in most non-cancer cells. The discrimination was based on the fact that targeted cells presented an increased signal due to the magnetic accumulation of CTCs. As a consequence, the increasing of SERS signal was correlated with the detection of CTCs (52).

Cancer biomarkers: circulating tumor molecular signature, from nucleic acids to proteins and exosomes. Cancer biomarkers proved to be extremely valuable for the diagnosis, prognosis and follow-up in oncology $(53,54)$. These molecules are released from the dead neoplastic cells in the bloodstream, becoming easily accessible to different detection methods. One of the most specific tumor markers is circulating tumor DNA (ctDNA), as it provides an exact insight with regards to tumor genotype.

Another type of genetic target for liquid biopsy is microRNA (miRNA). miRNA typically consists of a sequence of up to 25 nucleotides in a single strand of RNA. These particles are involved in gene regulation. Altered miRNA expression has been proven to be related to oncogenesis and disease progression (55). In comparison, miRNAs have a longer life in bloodstream and higher stability than ctDNA.

Nucleic acids can be detected by SERS $(56,57)$ even if there is a major limitation related to small amounts of cell-free DNA requiring high quality isolation and amplification methods. Recently, the detection of nucleic acids through SERS has been associated with polymerase chain reaction (PCR) for a better detection of ctDNA mutations $(58,59)$ by direct SERS. The approach consists of the following sequences. First, the desired analyte is identified based on a unique vibrational spectrum which is acquired upon its adhesion to the plasmonic surface. Noise reduction is obtained by minimizing the adsorbtion of other molecules and thus cancelling any signal overlap, by using spermine-coated silver colloids (AgSp) as a plasmonic substrate. In this manner intense and reproducible SERS spectra are generated even at low concentrations of nucleic acid. When analyzed, the spectra generated by the colloidal suspensions represent an authentic expression of the nucleic acid conformations (56).

This method proved to have a very high diagnostic potential even in the detection of clinically important punctual mutations in big oncogene fragments of K-ras. Consequently, it 
was successfully implemented in human assays, providing an efficient risk assessment tool for patients with prostate cancer, with a high sensitivity and specificity (60).

Recently, we have shown the correlation between DNA methylation pattern and SERS spectra, shedding light on a potential new method for cancer diagnosis $(31,61)$. It is well known that cancer DNA has a tendency towards hypomethylation. When DNA was extracted from an acute myeloid leukemia (AML) cell line, we found a decreased intensity of the $1005 \mathrm{~cm}^{-1}$ band of 5-methylcytosine compared to normal DNA, determined by the hypomethylation of the AML cells (31). This finding was then further tested on peripheral blood genomic DNA samples, yielding a high discriminative accuracy $(82 \%)$.

Expression of proteins is another useful method to detect cancer. The distribution of protein level provides information about the early diagnosis and prognosis (62). SERS analysis can also be applied for the detection of proteins, as for other biomarkers in cancer (44). Compared to the classic SERS approach, a new SERS hybrid detection method was developed for protein quantification, taking into account the high dimensions of these molecules $(63,64)$. Using this method, some elements from the surface of the molecules can be recognized by an active part of SERS that binds firmly the plasmonic surface and acts as an efficient transducer of signal by producing an intense vibrational signature.

The study of exosomes has gathered momentum in the recent years. The exosomes are extracellular vesicles with nanosized dimensions (20-150 nm) acting as intercellular signaling messengers. While analyzing their compositions, information regarding the cells and the microenvironment can be obtained (65). There are some advantages coming with the discovery of these exosomes, as they are found in a large amount in body fluids, they are more stable in circulation, easy to separate, handle and describe (65). As science is gaining a lot of information in the domain of tumor genesis and diagnosis using exosomes, research focused on the detection of exosomes using SERS approaches were described for the detection of CTCs and other tumor biomarkers (66-68).

SERS-based biofluid biopsy in CRC. The SERS detection of different oncologic biomarkers from biological liquids gained great interest in the last few years. In one study, the SERS intensity of many dominant vibrational bands of serum samples obtained from patients with CRC appeared increased, by comparison with the SERS serum spectra obtained from a healthy group (38). In another study, authors synthesized maghemite nanoparticles precoated with dimercaptosuccinic acid, which were used for targeting cells expressing characteristic carcinoembryonic antigen (CEA) of CRC (69). SERS was used to track the surface of nanosized maghemite particles from the beginning of the precoating up to the attachment of the anti-CEA fraction. Furthermore, when compared to control lines, the association with anti-CEA significantly increased the number of cells with maghemite nanoparticle internalization, as shown by transmission electron microscopy studies. The conclusion was that SERS can detect anti-CEA, being a potential theranostic tool for tumors which express it, micrometastasis, and cancer-circulating cells (69).
In the same field of tumor biomarker detection, another study has shown the feasibility of a SERS-based platform for quantitative analysis of CEA. The nanoparticles were prepared using anti-CEA-functionalized 4-mercaptobenzoic acid on a gold/silver core. Subsequently, CEA solutions at different known concentrations were analyzed in order to calibrate the platform. The method was further validated against the results obtained via electrochemical luminescence. The SERS-based model had a high sensitivity and specificity (70).

In a recent study, discrimination of CRC was achieved by using SERS with Ag hydrosol as substrate, coupled with principal component analysis-linear discriminant analysis (PCA-LDA). In order to differentiate between normal cells and those belonging to rectal cancer, SERS was applied with good results. In rectal cancer cells, there was a decrease in collagen (71) probably consequently to an increase in metalloproteinase activity. In another study using polarized SERS on Ag colloid substrate for the diagnosis of colorectal cancer from patient sera, authors reported a high diagnostic accuracy of SERS (91.6\%) (72).

Significant differences also arose when exosomes isolated from healthy cells were compared to CRC cells while using super-hydrophobic surfaces with nano-photonic surfaces for manipulation. Tumor exosomes had a higher RNA concentration and lower lipid vibration peaks (73).

In line with other studies, our research team showed great interest for the detection of cancer using SERS approaches (13). In a comprehensive study performed on breast, colorectal, lung, ovarian and oral cancer, an overall accuracy of $94 \%$ was achieved, based on SERS in conjunction with PCA-LDA. Regarding the CRC group, the sensitivity of cancer detection using SERS on patient sera was $83.3 \%$, with a specificity of $64.1 \%$ and a positive predictive value of $86.6 \%$. The best results were obtained on patient samples with ovarian cancer-sensitivity of $96 \%$ and specificity of $93 \%$.

Recently, our team published the results of a study performed with the aim of proving the diagnostic accuracy of SERS-based liquid biopsy in patients with gastric and colon cancer using a portable Raman device (11). There were significant differences in the SERS spectra between the cancer and control groups, especially with regards to carotenoids and purine metabolites (xanthine, hypoxanthine and uric acid). The cancer and control group were correctly discriminated with an accuracy of $76 \%$ based solely on their SERS profile. However, the accuracy was increased up to $83 \%$ by adding C-reactive protein, neutrophil and platelet counts and hemoglobin levels in the equation. A particular approach was the effort to translate our work in a clinical setting, using a portable Raman device, which is smaller and easier to accommodate in any laboratory setting, allowing for a cost and time efficient diagnosis (11).

\section{Conclusions}

SERS represents a promising tool for diagnosing, screening and following-up CRC patients. The key feature of the SERS method is the potential for very early detection, which might lead to a substantial improvement in the ultimate outcome: overall survival. Not least, there should be consequential gains 
with regards to quality of life and cost-efficiency, which can only increase along with further validation and calibration of the method. Of course, important caveats still remain, and questions regarding the discriminative powers and clinical application of SERS remain, as of now, with no definite answer. However, the first steps towards the introduction of portable Raman instruments in the clinical environment have already been made and the theoretical advantages have already been highlighted.

\section{Acknowledgements}

The authors would like to thank Dr. Vasile Coman for fruitful discussions and for the linguistic review.

\section{Funding}

AS and NL were supported by a grant from the Romanian Ministry of Research and Innovation, CCCDI-UEFISCDI, project number PN-III-P4-ID-PCCF-2016-0112 within PNCDI III and by the Competitiveness Operational Programme 2014-2020 (POC-A1-A1.1.4-E-2015), financed under the European Regional Development Fund, project number P_37_765. AS also acknowledges support from a grant offered by the Babeş-Bolyai University Cluj-Napoca, project number GTC 31370/2020.

\section{Availability of data and materials}

The datasets used and/or analysed during the current study are available from the corresponding author on reasonable request.

\section{Authors' contributions}

LA contributed to the conception and design of the study, data collection and writing the manuscript. AS had a major contribution in data collection and conception of the study. DC and NL contributed in the conception and design of the study, writing the manuscript, and final proofreading. VD contributed to the conception and design of the study and in data collection. EB contributed to the conception and design of the study and proofreading. RC and DA contributed to data collection, data analysis and writing the manuscript. IC contributed to the conception and design of the study and performed a critical review of the manuscript. All authors read and approved the final manuscript.

\section{Ethics approval and consent to participate}

The study was conducted in accordance with the Declaration of Helsinki. All patients mentioned in the part of 'personal results' signed the informed consent.

\section{Patient consent for publication}

Not applicable.

\section{Competing interests}

The authors declare that they have no competing interests.

\section{References}

1. Bray F, Ferlay J, Soerjomataram I, Siegel RL, Torre LA and Jemal A: Global cancer statistics 2018: GLOBOCAN estimates of incidence and mortality worldwide for 36 cancers in 185 countries. CA Cancer J Clin 68: 394-424, 2018.

2. Wolf AMD, Fontham ETH, Church TR, Flowers CR, Guerra CE, LaMonte SJ, Etzioni R, McKenna MT, Oeffinger KC, Shih YT, et al: Colorectal cancer screening for average-risk adults: 2018 guideline update from the American Cancer Society. CA Cancer J Clin 68: 250-281, 2018.

3. Brenner H, Stock C and Hoffmeister M: Effect of screening sigmoidoscopy and screening colonoscopy on colorectal cancer incidence and mortality: Systematic review and meta-analysis of randomised controlled trials and observational studies. BMJ 348: g2467, 2014.

4. Kim NH, Jung YS, Jeong WS, Yang HJ, Park SK, Choi K and Park DI: Miss rate of colorectal neoplastic polyps and risk factors for missed polyps in consecutive colonoscopies. Intest Res 15: 411-418, 2017.

5. Fernández-Lázaro D, García Hernández JL, García AC, Córdova Martínez A, Mielgo-Ayuso J and Cruz-Hernández JJ: Liquid biopsy as novel tool in precision medicine: Origins, properties, identification and clinical perspective of cancer's biomarkers. Diagnostics (Basel) 10: 215, 2020.

6. Shipp DW, Rakha EA, Koloydenko AA, Macmillan RD, Ellis IO and Notingher I: Intra-operative spectroscopic assessment of surgical margins during breast conserving surgery. Breast Cancer Res 20: 69, 2018.

7. Bourbousson M, Soomro I, Baldwin D and Notingher I: Ex vivo Raman spectroscopy mapping of lung tissue: Label-free molecular characterization of nontumorous and cancerous tissues. J Med Imaging (Bellingham) 6: 036001, 2019.

8. Boitor R, Kong K, Varma S, Koloydenko A, Williams H and Notingher I: Clinical translation of Raman-based multimodal spectral histopathology for margin assessment during surgery of basal cell carcinoma. Prog Biomed Optics Imaging Proc 11079: $1-4,2019$.

9. Zheng Q, Kang W, Chen C, Shi X, Yang Y and Yu C: Diagnosis accuracy of Raman spectroscopy in colorectal cancer: A PRISMA-compliant systematic review and meta-analysis. Medicine (Baltimore) 98: e16940, 2019.

10. Bonifacio A, Cervo S and Sergo V: Label-free surface-enhanced Raman spectroscopy of biofluids: Fundamental aspects and diagnostic applications. Anal Bioanal Chem 407: 8265-8277, 2015.

11. Avram L, Iancu SD, Stefancu A, Moisoiu V, Colnita A, Marconi D, Donca V, Buzdugan E, Craciun R, Leopold N, et al: SERS-based liquid biopsy of gastrointestinal tumors using a portable Raman device operating in a clinical environment. J Clin Med 9: 212, 2020.

12. Moisoiu V, Socaciu A, Stefancu A, Iancu DS, Boros I, Alecsa DC, Rachieriu C, Chiorean AR, Eniu D, Leopold N, et al: Breast cancer diagnosis by surface-enhanced Raman scattering (SERS) of urine. Appl Sci 9: 806, 2019.

13. Moisoiu V, Stefancu A, Gulei D, Boitor R, Magdo L, Raduly L, Pasca S, Kubelac P, Mehterov N, Chis V, et al: SERS-based differential diagnosis between multiple solid malignancies: Breast, colorectal, lung, ovarian and oral cancer. Int J Nanomedicine 14: 6165-6178, 2019.

14. Stefancu A, Moisoiu V, Couti R, Andras I, Rahota R, Crisan D, Pavel IE, Socaciu C, Leopold N and Crisan N: Combining SERS analysis of serum with PSA levels for improving the detection of prostate cancer. Nanomedicine (Lond) 13: 2455-2467, 2018.

15. Rusciano G, Capriglione P, Pesce G, Abete P, Carnovale V and Sasso A: Raman spectroscopy as a new tool for early detection of bacteria in patients with cystic fibrosis. Laser Phys Lett 10: 075603, 2013

16. Coman $\mathrm{C}$ and Leopold L: Raman mapping: Emerging applications. In Tech. February 15th, 2017. DOI: 10.5772/66097.

17. Fleischmann M, Hendra PJ and McQuillan AJ: Raman spectra of pyridine adsorbed at a silver electrode. Chem Phys Lett 26: 163-166, 1974.

18. Huang H, Shi H, Feng S, Lin J, Chen W, Huang Z, Li Y, Yu Y, Lin D, Xu Q and Chen R: Silver nanoparticle based surface enhanced Raman scattering spectroscopy of diabetic and normal rat pancreatic tissue under near-infrared laser excitation. Laser Phys Lett 10: 045603, 2013.

19. Huh YS, Chung AJ and Erickson D: Surface enhanced Raman spectroscopy and its application to molecular and cellular analysis. Microfluid Nanofluid 6: 285-297, 2009. 
20. Mircescu N and Colnita A: The intricate nature of SERS: Real-life applications and challenges 2017. In: Raman Spectroscopy and Applications. February 15th, 2017. DOI: 10.5772/65478.

21. Procházka M: Surface-enhanced raman spectroscopy: Bioanalytical, Biomolecular and Medical Applications. Springer, 2016.

22. Marks H, Schechinger M, Garza J, Locke A and Coté G: Surface enhanced Raman spectroscopy (SERS) for in vitro diagnostic testing at the point of care. Nanophotonics 6: 681-701, 2017.

23. Ding SY, You EM, Tian ZQ and Moskovits M: Electromagnetic theories of surface-enhanced Raman spectroscopy. Chem Soc Rev 46: 4042-4076, 2017

24. Otto A: Charge transfer in first layer enhanced Raman scattering and surface resistance. Quarterly Phys Rev 3: 2017.

25. Almehmadi LM, Curley SM, Tokranova NA, Tenenbaum SA and Lednev IK: Surface enhanced Raman spectroscopy for single molecule protein detection. Sci Rep 9: 12356, 2019.

26. Sha MY, Xu H, Penn SG and Cromer R: SERS nanoparticles: A new optical detection modality for cancer diagnosis Nanomedicine (Lond) 2: 725-734, 2007.

27. Stefancu A, Moisoiu V, Bocsa C, Bálint Z, Cosma DT, Veresiu IA, Chis V, Leopold N and Elec F: SERS-based quantification of albuminuria in the normal-to-mildly increased range. Analyst 143: 5372-5379, 2018.

28. Iancu SD, Stefancu A, Moisoiu V, Leopold LF and Leopold N The role of $\mathrm{Ag}^{+}, \mathrm{Ca}^{2+}, \mathrm{Pb}^{2+}$ and $\mathrm{Al}^{3+}$ adions in the SERS turn-on effect of anionic analytes. Beilstein J Nanotechnol 10: 2338-2345, 2019.

29. Leopold N, Stefancu A, Herman K, Tódor IS, Iancu SD, Moisoiu V and Leopold LF: The role of adatoms in chloride-activated colloidal silver nanoparticles for surface-enhanced Raman scattering enhancement. Beilstein J Nanotechnol 9: 2236-2347, 2018.

30. Stefancu A, Iancu SD, Moisoiu V and Leopold N: Specific and selective SERS active sites generation on silver nanoparticles by cationic and anionic adatoms. Rom Rep Phys 70: 509, 2018.

31. Moisoiu V, Stefancu A, Iancu SD, Moisoiu T, Loga L, Dican L, Alecsa CD, Boros I, Jurj A, Dima D, et al: SERS assessment of the cancer-specific methylation pattern of genomic DNA: Towards the detection of acute myeloid leukemia in patients undergoing hematopoietic stem cell transplantation. Anal Bioanal Chem 411: 7907-7913, 2019.

32. Psychogios N, Hau DD, Peng J, Guo AC, Mandal R, Bouatra S, Sinelnikov I, Krishnamurthy R, Eisner R, Gautam B, et al: The human serum metabolome. PLoS One 6: e16957, 2011.

33. Premasiri WR, Lee JC and Ziegler LD: Surface-enhanced Raman scattering of whole human blood, blood plasma, and red blood cells: Cellular processes and bioanalytical sensing. J Phys Chem B 116: 9376-9386, 2012.

34. Boyd S, Bertino MF, Ye D, White LS and Seashols SJ: Highly sensitive detection of blood by surface enhanced Raman scattering. J Forensic Sci 58: 753-756, 2013

35. Li S, Zhang Y, Xu J, Li L, Zeng Q, Lin L, Guo ZY, Liu Z, Xiong $\mathrm{H}$ and Liu S: Noninvasive prostate cancer screening based on serum surface-enhanced Raman spectroscopy and support vector machine. Appl Phys Lett 105: 091104, 2014

36. Li SX, Zhang YJ, Zeng QY, Li LF, Guo ZY, Liu ZM, Xiong HL and Liu SH: Potential of cancer screening with serum surface-enhanced Raman spectroscopy and a support vector machine. Laser Phys Lett 11: 065603, 2014.

37. Li SX, Zeng QY, Li LF, Zhang YJ, Wan MM, Liu ZM, Xiong HL, Guo ZY and Liu SH: Study of support vector machine and serum surface-enhanced Raman spectroscopy for noninvasive esophageal cancer detection. J Biomed Opt 18: 27008, 2013.

38. Lin D, Feng S, Pan J, Chen Y, Lin J, Chen G, Xie S, Zeng H and Chen R: Colorectal cancer detection by gold nanoparticle based surface-enhanced Raman spectroscopy of blood serum and statistical analysis. Opt Express 19: 13565-13577, 2011.

39. Casella M, Lucotti A, Tommasini M, Bedoni M, Forvi E, Gramatica F and Zerbi G: Raman and SERS recognition of $\beta$-carotene and haemoglobin fingerprints in human whole blood. Spectrochim Acta A Mol Biomol Spectrosc 79: 915-919, 2011.

40. Siravegna G, Marsoni S, Siena S and Bardelli A: Integrating liquid biopsies into the management of cancer. Nat Rev Clin Oncol 14: 531-548, 2017.

41. Zhang J, Chen K and Fan ZH: Circulating tumor cell isolation and analysis. Adv Clin Chem 75: 1-31, 2016.

42. Pallaoro A, Hoonejani MR, Braun GB, Meinhart CD and Moskovits M: Rapid identification by surface-enhanced Raman spectroscopy of cancer cells at low concentrations flowing in a microfluidic channel. ACS Nano 9: 4328-4336, 2015.
43. Yarbakht M, Nikkhah M, Moshaii A, Weber K, Matthaus C, Cialla-May D and Popp J: Simultaneous isolation and detection of single breast cancer cells using surface-enhanced Raman spectroscopy. Talanta 186: 44-52, 2018.

44. Zhang Y, Mi X, Tan X and Xiang R: Recent progress on liquid biopsy analysis using surface-enhanced Raman spectroscopy. Theranostics 9: 491-525, 2019.

45. Alix-Panabieres $\mathrm{C}$ and Pantel $\mathrm{K}$ : Challenges in circulating tumour cell research. Nat Rev Cancer 14: 623-631, 2014.

46. Shen Z, Wu A and Chen X: Current detection technologies for circulating tumor cells. Chem Soc Rev 46: 2038-2056, 2017.

47. Rawal S, Yang YP, Cote R and Agarwal A: Identification and quantitation of circulating tumor cells. Annu Rev Anal Chem (Palo Alto Calif) 10: 321-343, 2017.

48. Bhana S, Chaffin E, Wang Y, Mishra SR and Huang X: Capture and detection of cancer cells in whole blood with magnetic-optical nanoovals. Nanomedicine (Lond) 9: 593-606, 2014.

49. Sun C, Zhang R, Gao $M$ and Zhang $X$ : A rapid and simple method for efficient capture and accurate discrimination of circulating tumor cells using aptamer conjugated magnetic beads and surface-enhanced Raman scattering imaging. Anal Bioanal Chem 407: 8883-8892, 2015.

50. Alix-Panabieres $C$ and Pantel K: Clinical applications of circulating tumor cells and circulating tumor DNA as liquid biopsy. Cancer Discov 6: 479-491, 2016.

51. Cohen SJ, Punt CJ, Iannotti N, Saidman BH, Sabbath KD, Gabrail NY, Picus J, Morse MA, Mitchell E, Miller MC, et al: Prognostic significance of circulating tumor cells in patients with metastatic colorectal cancer. Ann Oncol 20: 1223-1229, 2009.

52. Shi W, Paproski RJ, Moore R and Zemp R: Detection of circulating tumor cells using targeted surface-enhanced Raman scattering nanoparticles and magnetic enrichment. J Biomed Opt 19: 056014, 2014.

53. Marrugo-Ramirez J, Mir M and Samitier J: Blood-based cancer biomarkers in liquid biopsy: A promising non-invasive alternative to tissue biopsy. Int J Mol Sci 19: 2877, 2018.

54. Haber DA and Velculescu VE: Blood-based analyses of cancer: Circulating tumor cells and circulating tumor DNA. Cancer Discov 4: 650-661, 2014.

55. Cheng G: Circulating miRNAs: Roles in cancer diagnosis, prognosis and therapy. Adv Drug Deliv Rev 81: 75-93, 2015.

56. Garcia-Rico E, Alvarez-Puebla RA and Guerrini L: Direct surface-enhanced Raman scattering (SERS) spectroscopy of nucleic acids: From fundamental studies to real-life applications. Chem Soc Rev 47: 4909-4923, 2018.

57. Laing S, Gracie K and Faulds K: Multiplex in vitro detection using SERS. Chem Soc Rev 45: 1901-1918, 2016.

58. Monroig-Bosque PC, Shah MY, Fu X, Fuentes-Mattei E, Ling H, Ivan C, Nouraee N, Huang B, Chen L, Pileczki V, et al: OncomiR-10b hijacks the small molecule inhibitor linifanib in human cancers. Sci Rep 8: 13106, 2018.

59. Koo KM, Wang J, Richards RS, Farrell A, Yaxley JW, Samaratunga $\mathrm{H}$, Teloken PE, Roberts MJ, Coughlin GD, Lavin MF, et al: Design and clinical verification of surface-enhanced Raman spectroscopy diagnostic technology for individual cancer risk prediction. ACS Nano 12: 8362-8371, 2018.

60. Guerrini L and Alvarez-Puebla RA: Surface-enhanced Raman spectroscopy in cancer diagnosis, prognosis and monitoring. Cancers (Basel) 11: 748, 2019.

61. Turcas C, Moisoiu V, Stefancu A, Jurj A, Iancu SD, Teodorescu P, Pasca S, Bojan A, Trifa A, Iluta S, et al: SERS-Based assessment of MRD in acute promyelocytic leukemia? Front Oncol 10: 1024, 2020.

62. Borrebaeck CA: Precision diagnostics: Moving towards protein biomarker signatures of clinical utility in cancer. Nat Rev Cancer 17: 199-204, 2017.

63. Feliu N, Hassan M, Garcia Rico E, Cui D, Parak W and Alvarez-Puebla R: SERS quantification and characterization of proteins and other biomolecules. Langmuir 33: 9711-9730, 2017.

64. Cheng L, Zhang Z, Zuo D, Zhu W, Zhang J, Zeng Q, Yang D, $\mathrm{Li} \mathrm{M}$ and Zhao Y: Ultrasensitive detection of serum MicroRNA using branched DNA-based SERS platform combining simultaneous detection of $\alpha$-fetoprotein for early diagnosis of liver cancer. ACS Appl Mater Interfaces 10: 34869-34877, 2018.

65. Barile L and Vassalli G: Exosomes: Therapy delivery tools and biomarkers of diseases. Pharmacol Ther 174: 63-78, 2017. 
66. Pang Y, Wang C, Lu L, Wang C, Sun Z and Xiao R: Dual-SERS biosensor for one-step detection of microRNAs in exosome and residual plasma of blood samples for diagnosing pancreatic cancer. Biosens Bioelectron 130: 204-213, 2019.

67. Yan Z, Dutta S, Liu Z, Yu X, Mesgarzadeh N, Ji F, Bitan G and Xie YH: A label-free platform for identification of exosomes from different sources. ACS Sens 4: 488-497, 2019.

68. Tirpe AA, Gulei D, Ciortea SM, Crivii C and Berindan-Neagoe I Hypoxia: Overview on hypoxia-mediated mechanisms with a focus on the role of HIF genes. Int J Mol Sci 20: 6140, 2019.

69. da Paz MC, Santos Mde F, Santos CM, da Silva SW, de Souza LB Lima EC, Silva RC, Lucci CM, Morais PC, Azevedo RB and Lacava ZG: Anti-CEA loaded maghemite nanoparticles as a theragnostic device for colorectal cancer. Int J Nanomedicine 7: 5271-5282, 2012
70. Chen G, Chen Y, Zheng X, He C, Lu JP, Feng S, Chen R and Zeng H: Surface-enhanced Raman scattering study of carcinoembryonic antigen in serum from patients with colorectal cancers. Appl Phys B 113: 597-602, 2013.

71. Li X, Yang T, Li S, Zhang S and Jin L: Discrimination of rectal cancer through human serum using surface-enhanced Raman spectroscopy. Appl Phys B 119: 393-398, 2015.

72. Lin D, Huang H, Qiu S, Feng S, Chen G and Chen R: Diagnostic potential of polarized surface enhanced Raman spectroscopy technology for colorectal cancer detection. Opt Express 24: 2222-2234, 2016

73. Tirinato L, Gentile F, Di Mascolo D, Coluccio ML, Das G, Liberale C, Pullano SA, Perozziello G, Francardi M, Accardo A, et al: SERS analysis on exosomes using super-hydrophobic surfaces. Microelectronic Engineering 97: 337-340, 2012. 